\title{
La institucionalización de políticas de protección de pueblos indígenas aislados: una mirada comparativa a Brasil y Ecuador
}

Ivette Vallejo Real*

\begin{abstract}
RESUMEN

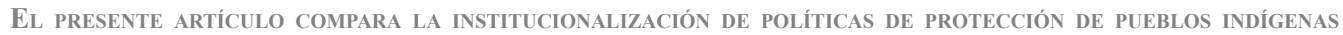
AISLADOS EN BRASIL Y ECUADOR. El PRIMERO, CON UNA LARGa TRAYECTORIA DE POLÍTICA INDIGENISTA Y El SEGUNDO, CON UNA ImplementaCión más ReCiente. En ambos países, abordamos la política pública en relación a PUEBLOS INDíGENAS AISLADOS Y LAS LIMITACIONES EXISTENTES PARA LA GARANTÍA DE SUS DERECHOS Y PROTECCIÓN. Esto en un contexto sudamericano -en la Amazonía y Gran Chaco- en que avanzan los frentes de CONTACTO AGROPECUARIO, MiNERO, PETROLERO, DE MEgA PROYECTOS DE DESARROLlO Y DE MONOCULTIVO, ASÍ COMO DE INTEGRACIÓN REGIONAL QUE GENERAN PRESIONES Y AFECTAN LA SOBREVIVENCIA FÍSICA Y CONTINUIDAD CULTURAL DE LOS PUEBLOS INDÍGENAS AISLADOS.
\end{abstract}

Palabras Claves: pueblos indígenas aislados - políticas de protección - derechos humanos.

\section{Abstract}

THIS ARTICLE COMPARES THE INSTITUTIONALIZATION OF PROTECTION POLITICS OF ISOLATED INDIGENOUS GROUPS in Brazil and Ecuador; one Country With a long history of, AND the OTHER WITH A MORE RECENT IMPLEMENTATION OF INDIGENOUS POLITICS. IN BOTH COUNTRIES, WE APPROACH PUBLIC POLICY IN RELATION TO ISOLATED INDIGENOUS GROUPS AND THE EXISTING LIMITATIONS TO GUARANTEEING THEIR RIGHTS AND PROTECTION. All of this in a South American context, -in the Amazonia and Gran Chaco-, where several frontiers of CONTACT ADVANCE, SUCH AS THE AGRicUlture, MiNing, OIL INDUSTRY, MEGa-DEVELOPMENT, MONOCULtURE AND REGIONAL INTEGRATION PROJECTS THAT AFFECT THE PHYSICAL SURVIVAL AND CULTURAL CONTINUITY OF THE ISOLATED INDIGENOUS GROUPS.

KEYWORDS: ISOLATED INDIGENOUS PEOPLE - PROTECTION POLITICS - HUMAN RIGHTS.

* Antropóloga. Doctora en Ciencias Sociales por la Universidad de Brasilia (UNB). Profesora-Investigadora de FLACSO sede-Ecuador del Departamento de Desarrollo, Ambiente y Territorio. Actualmente trabaja temas sobre extractivismo(s), territorios indígenas, conflictos socio-ecológicos, género y ambiente. Correo electrónico: ivallejo@flacso.edu.ec 


\section{Antecedentes}

$7 \mathrm{n}$ el mundo existen alrededor de 150 grupos indígenas aislados, 127 se encuentran en América del Sur, en la Amazonía, otro tanto en el Gran Chaco, y los demás en Asia, en las islas Andamán y Nicobar en el océano Indico, en Indonesia en Nueva Guinea (Loebens y Oliveira Neves, 2012).

Los Estados nacionales han incorporado territorios internos, en función de distintas coyunturas económicas y auges extractivos de conexión global, con el interés de la acumulación de capital, que ha generado periferización de sociedades, a la vez que geografías de la desigualdad (Wolf, 1982; Wallerstein, 1974). Diversos pueblos indígenas por distintas razones y procesos históricos, se han mantenido aislados o buscado zonas de refugio ante incursiones violentas. Se han utilizado varias denominaciones, "pueblos indígenas aislados", "pueblos indígenas en aislamiento voluntario", "pueblos ocultos", "pueblos libres", "sin contacto". Cabodevilla (2013) prefiere hablar de pueblos "ocultados, acorralados, desplazados" y cuestiona la idea de voluntariedad del aislamiento; considerándolo más como estrategia de supervivencia. Se manejará en este artículo la categoría de "pueblos indígenas aislados" (PIA), sobrevivientes de la violencia de distintos auges extractivos, de colonización y conquista.

Con el interés de analiza la situación de estos pueblos en Ecuador y Brasil, y la institucionalización de políticas de protección, intentaremos responder a algunas interrogantes planteadas en relación al tema: ¿qué marco legal e institucional se ha implementado para su protección?, ¿de qué manera las políticas precautelan la vida e integridad de los pueblos aislados y qué limitaciones existen?

En Brasil, la implementación de políticas de protección de PIA con la creación de entidades indigenistas se inspiró en lineamientos de carácter positivista. Más contemporáneamente en ambos países, Ecuador y Brasil, entidades de protección se orientan con lineamientos dirigidos a precautelar derechos humanos y evitar el etnocidio. Se conjugan derechos individuales y colectivos (autodeterminación, libre determinación, derechos territoriales, relacionados con identidad, entre otros) ${ }^{1}$. En estos países y otros de la región, -ya que varios PIA transitan entre fronteras-, desde un década atrás se han generado consensos para respetar su voluntad de aislamiento como un derecho, implementando estrategias de protección locales, transfronterizas y regionales (Brackelaire, 2006) 2 .

Sin embargo, más allá de estos marcos, desde una lectura crítica se puede evidenciar que la institucionalidad creada está impregnada por la colonialidad del poder (Quijano, 2000) ${ }^{3}$, y formas de bio-poder (Foucault, 1989, 2000) 4 o configuraciones de sociedades de control y disciplina, (De Negri y Hardt, 2001)5. En la tónica que han seguido las políticas y las entidades de protección de PIA se ejerce disciplinamiento que siguiendo a Foucault $(1989,2000)$ lo podríamos concebir

1 Si bien no existe a nivel del Sistema de Naciones Unidas (SNU) un marco específico sobre Pueblos Indígenas Aislados, la normativa sobre Derechos de los Pueblos Indígenas les abarca; por tanto, el Convenio 169 sobre Pueblos Indígenas y Tribales de la OIT (1998) y la Declaración de Derechos de los Pueblos Indígenas de Naciones Unidas (2007).

2 Recomendaciones del I Encuentro Internacional sobre Pueblos Aislados realizado en Belem, Pará (Brasil) en noviembre de 2005 por la Coordenação Geral de Indios Isolados - CGII de la FUNAI y el Centro de Trabalho Indigenista (CTI) que reunió la participación de especialistas y Defensorías del Pueblo de los países. En este encuentro se formó una Alianza Internacional para la protección de los Pueblos Indígenas Aislado y emergió la Declaración de Belem.

3 Por colonialidad del poder se entenderá aquellos rezagos heredados del proceso de la modernidad (eurocéntrica y capitalista), originada en los procesos de conquista del siglo XVI con continuidad a través de las configuraciones republicanas y posteriores de los Estados-naciones americanos.

4 Al hablar de biopoder, se toma como referencia la teoría foucaultiana que entiende que el poder funciona en términos de relación entre diferentes campos, instituciones, burocracias u otros grupos dentro del Estado. Poder que incita, induce, amplía o limita (Foucault, 1989, 2000).

5 De Negri y Hardt (2000), hacen referencia a tipos de sociedades que van acompañadas de instituciones que estructuran el terreno social; la meta del poder disciplinario sería producir seres humanos que pueden ser tratados como cuerpos dóciles. 
como una nueva anatomía política que impone sobre territorios y cuerpos (de PIA) una relación de docilidad-utilidad, que los explora, desarticula y recompone. Es así como, en la trayectoria indigenista en Brasil, se han utilizado tecnologías políticas de inserción de pueblos, y ajuste a procesos económicos, y en ambos países se nutren "sociedades de control" cuyos mecanismos se pueden ver envueltos en un cariz democrático, que se torna en razón de Estado'.

Las instituciones de protección, mediante una aguda vigilancia, o panoptismo, tienden a monitorear a los PIA con tecnologías sofisticadas para determinar dónde se encuentran, cuáles son sus áreas de asentamiento y movilidad; más que concentrarse de manera efectiva en detener el avance de los distintos frentes de las fronteras de contacto. Las políticas de protección quedan así, subordinadas a las prioridades del crecimiento económico. Cuestiones que serán tratadas a lo largo del artículo.

\section{Situación general de los pueblos indígenas aislados en Sudamérica}

Existen 127 referencias de PIA en América del Sur, en Amazonía y el Gran Chaco. De los 6 países donde se encuentran (Colombia, Ecuador, Perú, Bolivia, Brasil y Paraguay), Brasil tiene un total de 90 pueblos en la Amazonía7 (Loebens y Oliviera Neves, 2012), seguido de Perú, con alrededor de 20 PIA y otros de contacto inicial (Brackelaire, 2006), a lo largo de la frontera con Brasil y en diversos otros puntos (ver mapa 1).

En general continúa la ocupación de territorios, ampliación de frentes de conquista de la Amazonía y del Gran Chaco; ocurren genocidio-etnocidios encubiertos que amenazan con la extinción de los PIA, mientras pueblos de reciente contacto están en condiciones de extrema vulnerabilidad. En la Amazonía, los Estados buscan conectar la cuenca con el mercado globalizado, siguiendo la cartera de proyectos del Consejo Suramericano de Infraestructura y Planeamiento (COSIPLAN) de la Unión de Naciones Suramericanas (UNASUR) que conllevan a la implementación de la Infraestructura Regional Sudamericana (IIRSA), lo que seguramente ocasionará mayores impactos.

Varias amenazas pesan sobre los PIA en la región, en Perú están afectados por el extractivismo petrolero, carreteras, extracción forestal. La Amazonía está intervenida por el gran y pequeño capital nacional e internacional, y se favorecen a grandes proyectos de empresas madereras, petroleras y mineras (López y Miranda, 2012). En Colombia, las presiones de distintos actores (guerrilla, paramilitares, militares, narcotraficantes) relacionados con el conflicto armado han llevado a los Nukak Makú a desplazarse, y situarse precariamente en zonas periféricas del municipio de Calamar, en el departamento del Guaviare. En Bolivia y Paraguay la ampliación de la frontera agro-pecuaria, y la introducción de monocultivos genera presiones desde fincas y haciendas. En Paraguay, la mayor parte de tierras que son hábitat de los PIA están en manos de propietarios privados y las autoridades aprueban planes de uso productivo ganadero o forestal, sin considerar la presencia de pueblos aislados ayoreo (Brackelaire, 2006). En Bolivia el avance de madereras, haciendas y más recientemente la agroindustria de soya y caña amenaza a grupos

6 Foucault $(1989,2000)$ hace mención a una serie de instituciones de la modernidad y el capitalismo que se implementaron para ejercer una vigilancia perpetua del cuerpo social y constante de los individuos con fines de "normalización", con prácticas panópticas para observar movimientos, evaluar la conducta comparándola con la norma. El panoptismo sería una construcción de una anatomía política, que puede ser analizada cuando se trata a entidades para protección de pueblos aislados.

7 Las cifras son variadas: conforme plantea Mindlin (2015) al 2010, había en Brasil 305 pueblos indígenas, de ellos 60 o más eran aislados, la mayoría localizados en los bosques amazónicos. Actualmente, la Fundação Nacional o Indio (FUNAI) registra 78 pueblos aislados en Brasil, mientras el Conselho Indigenista Misionario (CIMI), habla de 90 .

8 La Cartera del COSIPLAN incluye 593 proyectos de integración, con inversión estimada de US\$182.436 millones, distribuidos en Suramérica. http://iirsa.org/News/Detail?Id=193 (23/03/2016). En Perú se apuntala el eje multimodal Manta-Belem que atravesaría la Reserva Territorial Napo-Tigre; la Transoceánica que atraviesa la frontera de Acre (Brasil) y Madre de Dios (Perú) y la carretera Cruzeiro do Sol (Brasil)-Pucalpa (Perú) que corta una región densa de grupos aislados. En Bolivia, vías presionarán pueblos aislados al atravesar el Gran Chaco. 


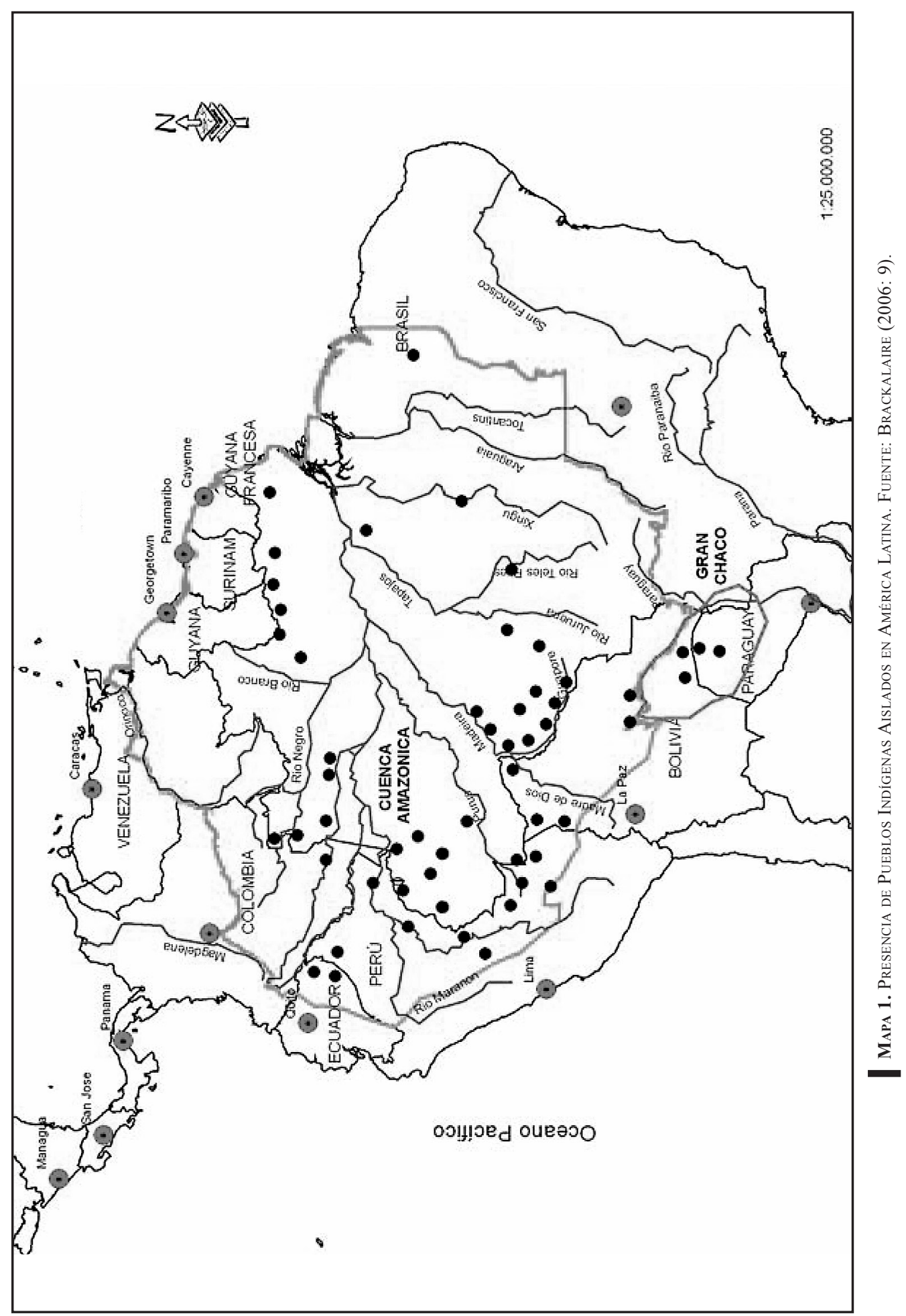


aislados ayoreo. Los Mbya- Yuqui están en proceso de contacto inminente por los parientes Yuqui evangelizados por la Misión Nuevas Tribus, y están presionados por madereros que invaden su territorio.

Existe una gradual invasión de territorios indígenas en tierras bajas por la expansión de la industria agrocomercial, exploración de recursos naturales, colonización de otras regiones (López y Miranda, 2012). En Venezuela si bien especialistas dicen que no hay PIA, algunos grupos Yanomami que permanecen en relativo aislamiento en la frontera con Brasil, transitan en la zona de las cabeceras del Orinoco. También hay grupos de Hoti (estado Amazonas) y de Sapé (estado Bolívar), pueblos en contacto inicial que necesitan acompañamiento especial (Brackelaire, 2006).

En cuanto al marco normativo en la región, en Perú no hay una referencia a PIA en la Constitución (1993), pero el Decreto Legislativo No. 28736 (de 2006) contiene la Ley para la protección de pueblos indígenas $\mathrm{u}$ originarios en situación de aislamiento y contacto inicial, y establece que debe garantizarse sus derechos de vida y salud, salvaguardar su existencia e integridad. Prohíbe actividades distintas a las de los usos y costumbres indígenas, pero deja la puerta abierta a proceder "de acuerdo a la ley" cuando la explotación de algún recurso sea de necesidad pública para el Estado.

En Bolivia, la Constitución (2009) reconoce de forma explícita derechos de PIA, así como la conservación ambiental de áreas habitadas por ellos. Se plantea la protección y respeto de sus formas de vida; que gozarán del derecho de mantener su condición de aislamiento, y que se delimite y consolide legalmente los territorios que habitan. Asimismo en la Constitución del Ecuador (2008), en el capítulo sobre Derechos de comunidades, pueblos y nacionalidades (artículo 57, No. 21), enuncia que los territorios de PIA son de posesión ancestral irreductible e intangible, en las que se vedará todo tipo de actividad extractiva. Se establece que el Estado adoptará medidas para garantizar sus vidas, respetar su autodeterminación y voluntad de permanecer en aislamiento; y que la violación de dichos derechos, constituirá delito de etnocidio. En consonancia, el Código Orgánico Integral Penal (COIP) legisla sobre los delitos de genocidio y etnocidio (Art. 79 y 80). En los tres países (Perú, Bolivia, Ecuador), estos avances normativos, no se han plasmado en la práctica para asegurar la protección de los PIA.

Al tratarse sobre políticas e instituciones creadas para la protección de PIA, Brasil ha sido una referencia en la región. En los demás países los esfuerzos desde el Estado han sido mínimos, o dispersos, con cierta actuación de las Defensorías del Pueblo (caso Colombia y Perú), o Fiscalía (caso Paraguay y Ecuador) y con una mayor agencia por parte de ONG's (caso del Perú y Paraguay). También la protección se ha dado a partir de la creación de unidades de conservación ambiental (tal es el caso de Colombia, Perú y Bolivia) ${ }^{9}$. Con estos antecedentes, trataremos a continuación la manera de cómo se han implementado políticas y marcos de protección de PIA en Brasil y Ecuador.

\section{Brasil entre políticas de tutela, integración y protección}

La matriz de formación colonial de Brasil ha estado históricamente marcada por el uso sistemático de la violencia contra los pueblos indígenas (Milanez, 2015). A lo largo del siglo $\mathrm{XX}$, la interiorización del desarrollo hacia el centro-oeste, norte del país y la Amazonía generó exterminio de varios pueblos; otros fueron llevados al límite de su existencia, sometidos por distintos actores interesados en sus territorios y en eliminar obstáculos para aprovechar suelos, recursos (minerales, forestales), o incorporarlos como mano de obra.

9 En Perú, la mayoría de PIA se encuentran en Áreas Naturales Protegidas, en Reservas Territoriales que corresponden a sus áreas de desplazamiento y que fueron establecidas para ello. En Colombia dentro del Parque Nacional Río Puré, la política de protección de PIA es inherente al texto de Resolución de la creación del parque (Brackelaire, 2006). 
En Brasil, en 1910 se creó el Serviço de Proteção aos Indios e Localização de Trabalhadores Nacionais (SPILTN), que en 1918 pasó a ser el Serviço de Proteção ao Indio (SPI) ${ }^{10}$; primera entidad indigenista regida por preceptos positivistas. En su visión, Brasil debía evolucionar, crecer en orden y progreso hacia la civilización para configurar una sociedad moderna (Milanez, 2015). Emergió el SPI de las actividades de la Comisión Constructora de la Línea Telegráfica, encargada de dar seguridad en las fronteras e integrar económicamente las regiones de Matto Grosso, Matto Grosso do Sul y Paraná.

Para unos, la entidad se encaminó a proteger a los indígenas, mientras para otros su finalidad era "pacificarlos para que fuesen gradualmente asimilados o integrados en la comunidad nacional" (Liebgott, 2012: 34) como mano de obra, a la vez que se conquistaban sus territorios. La política del SPI se direccionó a la "atracción" de los indígenas, utilizando regalos, establecimiento progresivo de contactos y "una rigurosa política de no violencia y prevención de conflictos" (Daiacon, 2002, en Milanez, 2015: 50). También utilizó intérpretes indígenas de los mismos troncos lingüísticos y confraternizaciones. Los funcionarios del SPI, provocaban situaciones de encuentro con indígenas que resistían al contacto, emprendían su pacificación, los sedentarizaban y constituían puestos indígenas (Ferreira, 2012), para sujetarlos a tutela federal.

El SPI tendría cambios en su política entre 1910 y 1967. En la década del 40 ocurrió un nuevo ciclo de auge del caucho durante la segunda guerra mundial, renovando anteriores impactos. Frente a ello, dentro de la entidad se nucleó el "sertanismo" para el trabajo con los PIA; función que se profesionaliza formalmente para los trabajadores de la Fundação Brasil Central (FBC), durante la época de la Marcha para el Oeste, decretada por Getulio Vargas. En la década habría dos líneas principales de actuación del indigenismo brasilero. Una de ellas, desde el SPI pretendía acelerar el contacto y la integración de los PIA para evitar el exterminio por parte de caucheros (seringalistas). Otra línea, desde la Fundacão Brasil Central (FBC) promovía una integración gradual, a lo largo de generaciones ${ }^{11}$; enfatizó en demarcar territorios continuos para uso exclusivo de indígenas, y garantizar la preservación cultural y ecológica' ${ }^{12}$.

La FBC tuvo acciones contradictorias. Dirigió expediciones en Roncador-Xingu, construyó pistas en el interior de Brasil y preparó la expedición Xingu-Tapajós hacia los años 50's. Se le cuestionó haber realizado transferencias de indígenas de otros lugares, hacia el Parque do Xingú ${ }^{13}$ y no haber frenado la expansión de actividades de pecuaria y ganadería.

Después de la segunda guerra mundial por lo menos 87 pueblos indígenas habrían sido llevados al exterminio ${ }^{14}$ por incursiones violentas de distintos frentes de expansión. Los frentes agrícolas se interesaban por los indígenas como mano de obra; los frentes pastoriles pretendían liberar territorios, para incorporar ganadería. En los 50, demarcar territorios para la protección de PIA era sustancial; sin embargo en la década del 60 hubo denuncias de corrupción, y de que en las instalaciones de los puestos del SPI se estaba produciendo exterminio, envenenamientos y torturas. Las denuncias a escala internacional de la ocurrencia de genocidio y las omisiones del SPI en prevenir masacres ${ }^{15}$, llevaron a dimitir funcionarios, suspender a esta entidad y crear la Fundação Nacional do Indio (FUNAI) en 1967.

10 El enfoque de la "localización de trabajadores nacionales" sería transferido al Ministerio de Negocios, Agricultura, Industria y Comercio, concentrándose el SPI en indígenas desde 1918.

11 El lineamiento de integración rápida era propuesto por Chico Meireles del SPI; mientras el de la integración gradual y lenta propuesta por los hermanos Villas Boas.

12 Con esta idea se creó el Parque Indígena do Xingu en 1961 en un contexto en que varios pueblos de la zona experimentaban epidemias y acoso por la expansión violenta agro-pastoril de las élites económicas y políticas del Estado de Mato Grosso.

13 Un caso dramático fue la transferencia de los indígenas Panará al Xingu. Su territorio fue cortado por la construcción de la carretera BR-163 de Cuiabá a Santarém, que pasó por sus aldeas. Los militares incentivaban el contacto por el interés de minerales (oro, diamante). La construcción de la vía generó epidemias de gripe y neumonía. En menos de 3 años, casi murió todo el pueblo. En 1974 los sobrevivientes fueron transferidos al Xingú.

14 Si para 1900 "existían 105 grupos indígenas aislados, con una población de 50.000 personas. En 1957 "se estimaba una reducción poblacional en casi 75\%", (Milanez, 2015: 50).

15 Como lo sucedido en el rio Jamanxim, que afectó a los indígenas Pakaã-Nova y Xavante. 
La FUNAI durante la égida de gobiernos civil-militares tomó control de territorios con recursos mineros, implementó infraestructura de desarrollo, y direccionó esquemas de colonización, sobrepuestos a la protección de PIA ${ }^{16}$. En la década del 70 a partir de la aprobación del Estatuto do Indio (1973) se ampliaron lineamientos de protección y derechos territoriales, pero ocurrieron masacres contra PIA conforme avanzaban las políticas de desarrollo e integración de la Amazonía ${ }^{17}$.

\section{Creación de una entidad para pueblos indígenas aislados en Brasil}

A fines de la década de los 80, la democratización de Brasil incidió en cambios en la política indigenista ${ }^{18}$, sin embargo varias masacres quedaron impunes ${ }^{19}$. En 1987 se creó el Departamento de Índios Isolados (DEII) dentro de la FUNAI (Milanez, 2015) y entre 1991 y 1993, durante el gobierno de Collor de Mello, en lo posterior a la Constituyente hubo un proceso intenso de demarcación de tierras indígenas.

Entre las acciones del DEII se crearon Frentes de Protección Etnoambiental (FPE) responsables de realizar sobrevuelos aéreos, monitorear los ríos para identificar amenazas sobre PIA, localizar malocas y chacras (roças), mapear y dimensionar las tierras que ocupan. En el 2000 se establecieron directrices de protección entre ellas, garantizar el no contacto de PIA; asegurar su autonomía y libertad; creación de programas de localización; impedir la ocupación de sus territorios; garantizar su integridad; preservar sus ecosistemas; fiscalizar y prohibir actividades económicas o comerciales al interior de sus tierras por parte de terceros (Liebgott, 2014). Si bien el DEII actuaba con estos lineamientos, el Instituto Nacional de Colonização e Reforma Agraria (INCRA) permitía colonizaciones y explotación forestal en tierras indígenas.

En el 2009 fue constituida la Coordinação Geral de Indígenas Aislados y Recem Contactados (CGGII) en reemplazo del Departamento de Indios Isolados (DEII), y en las reformas administrativas de la FUNAI de 2009 al 2012, desapareció la palabra "sertanista", cuyas funciones pasaron a ser ejercidas por funcionarios de los FPE en Maranhão, Amazonas, Pará y Acre.

\section{Nuevas presiones sobre pueblos indígenas aislados de Brasil}

En 2007 el gobierno de Dilma Rouseff lanzó el Programa de Aceleração do Crecimento (PAC), vinculado a IIRSA ${ }^{20}$ que incluye hidroeléctricas, hidrovías y grandes carreteras. De 114 emprendimientos propuestos, 42 afectarían regiones con presencia de PIA (Milanez, 2015).

16 De esa época, se ha cuestionado que funcionarios de la FUNAI junto con el Instituto Brasileiro de Medio Ambiente (IBAMA) y después el INCRA promovieran acuerdos con segmentos políticos y económicos regionales patrocinando la invasión de tierras para explotación maderera y minera, y la formación de latifundios en la Amazonía, con finqueros provenientes del centro-sur del país (Liebgott, 2014). Desde el Estado se subvencionó a latifundistas, cambiando la lógica previa de estimular el asentamiento de trabajadores rurales.

17 Durante la dictadura militar la construcción de las hidroeléctricas de Balbina y Tucurui, y las carreteras Transamazónicas (Belem-Brasilia /BR 364; BR 174 y la perimetral norte) y la Transoceánica (Brasil-Perú) que atravesaron la Amazonía, produjeron invasiones en territorios indígenas que "diezmaron los pueblos WairimiAtroari, Yanomami, Arara, Parakanã, Cinta Larga, Nambikwara, entre otros, llevándolos al borde del exterminio" (Loebens, 2014: 29).

18 En los años subsiguientes a la promulgación de la Constitución Federal (1988) se reconoció a los indígenas como portadores de derechos diferenciados. Se avanzó en procesos demarcatorios, se firmó decretos que traspasaron competencias en salud y educación de la FUNAI hacia ministerios del ramo.

19 Casos de masacre de los pueblos Kanoê y Akuntsu ocurrieron en la región de Corumbiara, al sur de Rondonia en la década del 80, y remanentes fueron contactados por la FUNAI en 1995.

20 IIRSA emergió del encuentro mantenido entre presidentes de América Latina en Brasilia en el 2000. Articularía infraestructura de transporte, energía, comunicación; crearía nuevos corredores de exportación y flujo de mercaderías para alcanzar mayor competitividad en mercados mundiales. Incluye 510 proyectos en 47 planes y 10 ejes modales de integración latinoamericana a ser financiados por BID, CAF, BNDES y BM (López y Miranda, 2014). 
Habrían nuevas estrategias de control de recursos naturales, apertura de nuevas carreteras, construcción de hidroeléctricas grandes y pequeñas, gaseoductos, explotación de petróleo y gas, incentivos para la agroindustria y agricultura a gran escala y planes de minería. En últimos años se flexibilizó la normativa ambiental con un nuevo Código Forestal y un Código de Minería. También, propuestas legislativas con influencia de grupos de poder económico que pretenden transferir al Congreso Nacional las responsabilidades de la FUNAI en demarcar tierras indígenas.

En el 2009 se expidió el Decreto 7056 que pretendía reestructurar la FUNAI. Se crearían 6 nuevos Frentes de Protección Etnoambiental, totalizando 12 unidades para el trabajo con PIA y pueblos de reciente contacto; sin embargo al 2010 la FUNAI apenas había gastado 36\% de los recursos presupuestados en este sentido.

Existen en la CGGII innovaciones tecnológicas, bancos de datos, uso de GPS para registro georeferenciado y metodologías de trabajo con PIA para el levantamiento de vestigios de ocupación indígena. En la práctica no obstante, se cuestiona que la entidad avale proyectos económicos vinculados con el Programa de Aceleración del Crecimiento (PAC), que afectan recursos naturales de la región, a la vez que reducen territorios que son refugio de PIA(Milanez, 2015; López y Miranda, 2012) ${ }^{21}$. Entre los proyectos ligados a IIRSA la pavimentación de la BR-364 y la conclusión de la carretera del Pacífico, habilitarán la extracción forestal y prospección petrolera.

Ambientalistas, indigenistas y organizaciones indígenas han efectuado denuncias con relación a la concesión de licencias para las grandes hidroeléctricas (Santo Antonio y Jirau en el rio Madeira en Rondonia en construcción, y Belo Monte, en el río Xingu en Pará) por el impacto que tendrán sobre los PIA, sus prácticas culturales, movilidad territorial y formas de aprovisionamiento ${ }^{22}$. El complejo hidroeléctrico sobre el río Madeira, principal río tributario del Amazonas impactará a alrededor de 15 PIA en Rondonia, al inundar su territorio. Además está prevista la ampliación de navegación más arriba de la ciudad de Porto Velho, a través de los ríos Orthon, Madre de Dios, Beni, Mamoré y Guaporé completando una hidrovía de Porto Velho (Rondonia) hasta Itacoatiara en Amazonas.

Muchos grupos aislados para mantener su autonomía se dislocan hacia áreas más preservadas, algunas de ellas son tierras indígenas demarcadas a específicos pueblos, produciéndose disputas territoriales, como sucede en la frontera entre Acre y Perú. En otros casos a pesar de que los PIA estén en territorios demarcados para otros pueblos indígenas, reciben presiones conforme avanza la exploración ilegal de madera. En ciertos casos el gobierno federal en vez de demarcar tierras para PIA, creó Unidades de Conservación que no necesariamente contienen las amenazas de actores externos.

Las acciones de localización, monitoreamiento y vigilancia de la Coordinação Geral de indios Isolados e de Recente Contacto (CGIIRC) son insuficientes a la dinámica de sobreposición de los frentes de presión (infraestructura estatal, mega extractivismo de recursos naturales), y a la extracción ilegal de madera, especulación de tierras, minería (garimpo), caza y pesca ilegal.

21 En la faja que se extiende por toda la región de frontera del estado de Acre con Perú, se encuentra una de las mayores áreas de movilidad de PIA. A partir del gran corredor regional que se formó entre los frentes de expansión económica entre Brasil, Perú y Bolivia, los PIA se refugiaron en áreas de cabecera de grandes ríos y sus afluentes entre el Ucayali, Juruá, Purus, Javari y Madre de Dios; al ser áreas de difícil acceso, ríos encajonados y donde no hay productos de interés comercial (caucho, castaña u otros). Habría sido zona de refugio en el auge cauchero para varios pueblos y en últimas décadas refugio de pueblos que en el Perú huyen de carreteras, explotación maderera, de petróleo y gas natural.

22 Señala Loebens (2014) que a pesar de que la FUNAI dirigió estudios en el río Madeira reconociendo la existencia de 5 grupos aislados en el área de influencia de la hidroeléctrica de Belo Monte, la entidad avaló su construcción. 


\section{Pueblos indígenas aislados en el Ecuador}

En Ecuador existen PIA en las provincias de Orellana, Pastaza y Napo, incluyendo el Parque Nacional Yasuní (PNY) y la frontera con el Perú. Se movilizan en zonas entre los ríos Tiputini, Curaray, Tivacuno, Yasuní, Nashiño, Cononaco, Cononaco Chico, Tiwino y Cuchiyacu. Se menciona 4 grupos de PIA: Tagaeri en el Parque Nacional Yasuní, Zona Intangible; Taromenane entre los ríos Tiputini, Nashiño y Cononaco en el Parque Nacional Yasuní, Zona Intangible en las provincias de Orellana y Pastaza frontera con Perú; un grupo aislado Waorani en los ríos Yasuní, Nashiño, Cononaco en Orellana y Pastaza (frontera con Perú) y aislados posiblemente del grupo Zaparoano entre los ríos Napo y Tigre, Pastaza en la frontera con el Perú (Loebens y Oliviera Neves, 2012; Berraondo y Cabodevilla, 2005) ${ }^{23}$.

Sobre los Taromenane, no se tiene claridad si provendrían del mismo tronco lingüístico waorani; se considera son más lejanos en parentesco a los waorani y también se los asocia con grupos Sapaorani, Sapara o Muratos (Berraondo y Cabodevilla, 2005), cuyo territorio de movilidad y ocupación está dentro del Parque Nacional Yasuní. Conforme a los waorani "se trata de un grupo desemejante del propio. Distinto pero similar, otro pero igual" (Proaño y Colleoni, 2008: 50). Han mantenido con ellos relaciones de enfrentamiento "hace cien años atrás" (Lopez y Miranda, 2012: 219). Cabodevilla (1990) considera que es un grupo waorani separado de los demás a inicios del XX, que habrían dejado su territorio tradicional en la frontera con el Perú, para remontarse hacia las cabeceras de los ríos Yasuní y Tiputini. Hacia el 2003 se tenía referencias de grupos Taromenane ocupando las cuencas de los ríos Tiputini, Nashiño y Cononaco (López y Miranda, 2012).

Con respecto a los Tagaeri, se considera estarían emparentados con waorani a través de los clanes Baihuairi y Peyemoiri. Comparten territorio ancestral que incluye la Zona Intangible ${ }^{24}$. A las familias waorani en aislamiento se las ubica en la zona del alto Yasuní, y con una movilización entre Ecuador y Perú (Berraondo y Cabodevilla, 2005). En el caso de los waorani (en sus varios grupos familiares-clanes) se trata de un pueblo de reciente contacto desde la injerencia del Instituto Lingüístico de Verano (ILV) en la década del 50, en el siglo anterior y que ha vivido un proceso de transformaciones aceleradas, de reducción de su territorio de ocupación que abarcaba desde el río Napo al Curaray, así como el traslape de bloques de explotación petrolera. Situación acompañada de la ampliación de distintos frentes: construcción de ejes viales, extracción forestal, turismo no controlado, entre otros aspectos.

El Parque Nacional Yasuní (PNY), en la Amazonía ecuatoriana que alberga a estos distintos grupos $^{25}$, se extiende en las cuencas de los ríos Yasuní, Cononaco, Nashiño y Tiputini. En el presente siglo, han ocurrido dos eventos de masacres de Tagaeri y Taromenane: el primero, en el 2003 fue liderado por los babeiri (grupo de Babe) waorani ${ }^{26}$; y el segundo, en 2013 fue cometido por wepeiri (gente de Wepe) ${ }^{27}$ waorani, llevando a grupos PIA al borde de la extinción, con el asesinato principalmente de mujeres y niños.

23 Además de los pueblos Tagaeri y Taromenane autores como Smith (1996, citado en Amicus Curiae sobre las medidas cautelares MC-91-06 otorgadas en favor de los pueblos indígenas Tagaeri y Taromenane en situación de aislamiento, Ecuador, Colectivo de Antropólogas del Ecuador, octubre/ 2013), mencionan Huiñitairi, Oñamenane y posiblemente otros grupos que cruzan la frontera de Perú-Ecuador y se movilizan en las zonas del bajo Curaray y Nashiño.

24 ZIIT declarada por decreto presidencial en 1999, que además forma parte del PNY.

25 El PNY fue creado en julio de 1979 por Decreto Inter-Ministerial No. 322. Sus límites fueron modificados en 1990 y en 1992, cubriendo finalmente una superficie de 982.000 ha. Fue declarado como Reserva de Biósfera por la UNESCO, en 1989

26 La matanza ocurrió en mayo de 2003 dentro de la ZITT establecida por el Estado en 1999. Según Proaño y Colleoni (2008) murieron 15 mujeres y niños Tagaeri-Taromenane.

27 Para referirse a los Wepeiri, Proaño y Colleoni (2008) los conciben como "aucas de las colinas" para identificar a los grupos waorani que vivían antes del contacto entre los ríos Tiputini y Cononaco. 


\section{Declaratoria de una zona intangible en el Yasuní}

En 1998 el Ministerio de Ambiente (MAE), Energía y Minas y el Consejo de Desarrollo de las Nacionalidades y Pueblos del Ecuador (CODENPE) conformaron una Comisión Interministerial para impulsar la declaratoria de zonas intangibles. En 1999 se declaró una Zona Intangible de 700 ha. en el núcleo del PNY para que Tagaeri y Taromenane queden "protegidos a perpetuidad de cualquier actividad minera, petrolera y colonización" ${ }_{28}$. Se enunció en el Decreto 552 , se garantizará su derecho a no ser desplazados de sus tierras, conforme lo establecido en la Constitución de 1998 (Art. 84); se estableció tutela jurídica dirigida a "la protección de las culturas ancestrales selváticas y la posibilidad de desenvolverse conforme a sus propios valores y tradiciones". Se marcó que la Zona intangible Tagaeri-Taromenane (ZITT), se ubicaría en las parroquias de Cononaco y Nueva Rocafuerte, cantón Aguarico en la provincia de Orellana y en la parroquia de Curaray, cantón Pastaza, en la provincia de Pastaza, y que sus límites se definirían en el lapso de 120 días; sin embargo, "permaneció sin delimitarse, olvidada de los poderes públicos desde 1999 hasta agosto de 2004" (Melo, en Proaño y Colleoni, 2008: 14).

Al 2004 mediante Acuerdo Inter-ministerial 092 los Ministerios del Ambiente y de Energía y Minas constituyeron una Comisión para la Delimitación de la ZITT y suscribieron una carta de entendimiento entre el Ministerio del Ambiente, WCS, el proyecto CAIMAN y EnCanEcuador S.A para realizar un estudio técnico. La Comisión recomendó una extensión de 783.213, 21 ha. para la ZITT29. Según el informe se quería "minimizar la fragmentación de bloques petroleros, siempre y cuando no implique una amenaza directa a las zonas de desplazamiento y ocupación de los clanes Tagaeri y Taromenane, especialmente a lo largo de la cuenca del río Nashiño" ${ }_{30}$.

Los estudios técnicos encomendados y la Comisión Interministerial conformada respondían a la difusión internacional de la matanza del clan taromenane ocurrida en el 2003. Se considera que en ella hubo incitación de parte de madereros ilegales interesados por la extracción de maderas finas, principalmente cedro, en los territorios de Tagaeri y Taromenane, lo que se habría sumado al interés de venganza del grupo de Babe ${ }^{31}$. La extracción forestal ilegal, se reportaba en las zonas aledañas a los ríos Shiripuno y Tiwino ubicados al sur de la vía Auca. Habría también intervenciones madereras en el curso del río Cononaco y aguas arriba del río Cachiyacu (Ecociencia, 2004).

Al 2005 se preveía que la licitación del bloque ITT y del proyecto del bloque 31 incrementaría los niveles de impacto directo ya existentes en el bloque 16, ya que generaría un polo de atracción que motivaría migraciones de población waorani hacia el límite norte de la ZITT.

28 Tomado de Ministerio de Medio Ambiente y Unión Europea. Zonas intangibles de la Amazonía Ecuatoriana, Ministerio de Medio Ambiente, Ministerio de Energía y Minas, CODENPE, PETROECUADOR y Proyecto PETRAMAZ de la Unión Europea, 1999.

29 Los límites propuestos por la comisión se solapaban con porciones de los bloques petroleros: 17 adjudicado entonces a EnCanEcuador S.A (48,6\%), con bloque 31 adjudicado a PetroEcuador $(11,6 \%)$ y con el bloque denominado para entonces 20 (ITT) administrado por PetroEcuador (11,6\% del bloque). Establecía el informe que la ZITT presentaba un 3 er. traslape con el segmento sur del bloque ITT. Decía que precautelar esta zona protegería formaciones aluviales de la cuenca del río Nashiño con formaciones de moretales que eran reservas de cacería estacional para el clan Taromenane. Se enunciaba tener evidencias de que en la estación de estiaje, coincidente con la maduración de frutos dicha palmácea generaba movilidad de animales, por lo que constituía un corredor natural y de "vital importancia para los desplazamientos de las poblaciones Taromenane o Taromenga" (Estudio técnico para la delimitación de la zona intangible Tagaeri-Taromenane en el territorio Étnico Huaorani y Parque Nacional Yasuní" Informe de WCS, 2005: 14). El estudio técnico recomendaba consolidar la ZITT, fijar definitivamente sus linderos y coordenadas de bloques petroleros, resolver traslapes con bloques 17 y 31; y desarrollar un Plan de Protección y Defensa de los clanes waorani en aislamiento voluntario que se encuentren al interior de la ZITT, establecer un régimen especial para garantizar su protección y derechos.

30 Tomado de "Estudio técnico para la delimitación de la zona intangible Tagaeri-Taromenane en el territorio étnico huaorani y Parque Nacional Yasuní", Informe presentado por WCS a los Ministerios del Ambiente y Energía y Minas, 2005.

31 La tala ilegal instalada en zonas de refugio de Tagaeri -Taromenane habrían ocasionado encuentros violentos entre madereros e indígenas, una década antes. 


\section{Un Plan de Medidas Cautelares y una instancia de protección}

En el 2006, la Comisión Interamericana de Derechos Humanos otorgó medidas cautelares a favor de los PIA, a partir de la acción colectiva emprendida ante este organismo por un grupo de ciudadanos ${ }^{32}$. Se "solicita al Estado ecuatoriano que adopte medidas efectivas para proteger la vida e integridad personal de los miembros de los pueblos Tagaeri y Taromenane" (Melo en Proaño y Colleoni, 2008: 14). Posteriormente, el 3 de enero de 2007 por Decreto Ejecutivo No. 2187, durante el gobierno de Palacio, se delimitó la ZITT con 758.051 ha. ubicadas en las parroquias de Cononaco y Nuevo Rocafuerte del cantón Aguarico, provincia de Orellana y en la parroquia Curaray del cantón Arajuno, provincia de Pastaza ${ }^{33}$.

En octubre de 2007, al inicio del gobierno de Rafael Correa, se suscribió el Acuerdo Interministerial No. $033^{34}$ para crear una estructura institucional responsable de la implementación del Plan de Medidas Cautelares para Pueblos en situación de Aislamiento Voluntario (PMC), y en enero de 2008 se creó una Unidad Técnica del Ministerio de Ambiente (MAE) adscrita al Programa de Reparación Ambiental y Social (PRAS). Entre sus líneas de acción constaría: coordinar y ejecutar acciones de monitoreo de la ZITT con levantamiento de información sobre la localización y desplazamientos de los PIA y sobre presiones efectivas o potenciales que les afecten. Se instaló un puesto de control en el puente del río Shiripuno para frenar por vía terrestre y fluvial actividades ilegales en la ZITT, se efectuó un programa de capacitación de militares y policías, se contrató un equipo técnico e incluyó monitores indígenas waorani, (Proaño y Colleoni, 2008).

Otras actividades, como un sistema de vigilancia epidemiológica con una red comunitaria de promotores de salud, el impulso de alternativas de subsistencia para la población waorani, no tuvieron mayor avance. Dentro del PMC se estableció la responsabilidad de coordinar acciones entre ministerios (Gobierno, Ambiente, Salud, Relaciones Exteriores, Defensa, y Coordinador de Patrimonio Cultural). Otras acciones asumidas sería la veda forestal por Decreto Ejecutivo No. 419 de junio de 2007 para fortalecer el control en el PNY ${ }^{35}$; y se estableció un Código de Conducta de Operaciones Hidrocarburíferas en zonas de movilidad de pueblos aislados (Melo, en Proaño y Colleoni, 2008).

Pese a la creación de una ZITT y la implementación del PMC, ocurrieron muertes y ataques violentos, lo que ha mostrado que las medidas adoptadas no han sido suficientes ${ }^{36}$. La zona que rodea a la ZITT en la provincia de Orellana está atravesada por vías de acceso y las carreteras, principalmente la vía Auca que corta en dos al territorio waorani y de los pueblos aislados (Proaño y Colleoni, 2008). Además, desde el Estado se ha fomentado la colonización de kichwas y shuar, creando un escenario de tensiones. En el área para el 2008 había "cuatro bloques petroleros, tres campos marginales y numerosos pozos en producción" (Proaño y Colleoni, 2008).

El 18 de Octubre de 2010, mediante Decreto Ejecutivo No. 503 (Registro Oficial No. 302), se transfirieron las competencias del PMC del Ministerio del Ambiente, al Ministerio de Justicia y Derechos Humanos ${ }^{37}$ y se prosiguió en actividades de monitoreo. Una nueva matanza de PIA ocurriría en el $2013^{38}$ como retaliación al lanceamiento de dos ancianos waorani de Yarentaro (bloque 16); nuevamente la efectividad del PMC se puso en entredicho.

32 Un grupo de biólogos y abogados emprendió dicha acción en vista de rumores de la ocurrencia de una nueva masacre de aislados en el Shiripuno.

33 La delimitación tuvo menos $25.162,21$ ha., que la propuesta anterior.

34 Suscrito entre el Ministerio Coordinador de Patrimonio Natural y Cultural, Ministerios de Ambiente, y de Minas y Petróleo.

35 Decreto firmado entre el Ministerio de Ambiente, de Defensa y Policía Nacional.

36 Se produjeron dos ataques mortales en 2008 y 2009 por parte de "pueblos ocultos" (Taromenane) en las cercanías de Armadillo y la zona de Hormiguero (Cabodevilla, 2013). En el 2008 murió lanceado cerca de Armadillo un maderero colono quien trabajaba en colaboración con un wao de Ñoneno. Al 2009 en Los Reyes murieron una señora colona y dos de sus hijos.

37 Dicho ministerio devendrá en Ministerio de Justicia, Derechos Humanos y Cultos en junio de 2010 por disposición del ejecutivo.

38 En marzo de 2013 la pareja de ancianos waorani Ompure y Buganey de la comunidad de Yarentaro (bloque 16) fueron lanceados y poco después el 29 de marzo de 2013 ocurrió la matanza de un grupo de indígenas Taromenane perpetrada por familiares de los ancianos. 
El presidente de la República por Decreto Ejecutivo 17, del 6 de octubre de 2013, creó una Comisión para investigar de forma diacrónica las muertes y las disputas entre los pueblos indígenas Waorani y Taromenane; la misma debía presentar recomendaciones para fortalecer la protección de los PIA. La Comisión estaría adscrita a la Secretaría Nacional de Gestión de la Política y sería presidida por el Fondo Ecuatoriano Populorum Progressio (FEPP), una ONG articulada a la iglesia católica, con experiencia de trabajo en la Amazonía en Orellana y Pastaza. En la Comisión participarían también técnicos del Ministerio de Justicia, Derechos Humanos y Cultos. La Comisión culminó su trabajo en junio de 2014 y recomendó la creación de una Dirección de Protección de Pueblos indígenas en Aislamiento Voluntario y Contacto Inicial (DPPIAV), que estaría adscrita al Ministerio de Justicia Derechos Humanos y Cultos. La Dirección propuesta, intervendría ante emergencias, coordinaría un trabajo interinstitucional y propiciaría una cultura de paz en el Yasuní.

\section{Lineamientos de protección y contención de la violencia en el Yasuní}

Del 2008 al 2016 si bien se ha institucionalizado una política de protección de PIA en el Ecuador, no se han logrado acciones integrales que prevengan enfrentamientos entre Waorani-Tagaeri-Taromenane. Durante la gestión del PMC en el Ministerio de Justicia, no hubo una acción contundente para evitar la matanza de PIA perpetrada como venganza en el 2013 por familiares de las comunidades de Dikaro y Yarentaro y allegados indirectos de los ancianos muertos. El PMC no ofreció compensaciones oportunas a los familiares (Cabodevilla, 2013) ${ }^{39}$, no se activó un Plan de Contingencia ${ }^{40}$ ni se condujo un proceso de pacificación y contención" (Alvarez, et al., 2016). También se evidenció la falta de coordinación inter-institucional entre el PMC, el Ministerio de Justicia, con Fiscalía y otros Ministerios. Posterior a la matanza, si bien se efectuaron investigaciones desde la Fiscalía General del Estado, que incluyó un peritaje antropológico, las acciones de administración de justicia han resultado contraproducentes ${ }^{41}$.

Se teme hayan ocurrido nuevas incursiones de Waorani a clanes PIA, no reveladas. Asimismo, recientemente, ocurrieron nuevos hechos de muerte, que evidencian que los PIA lancean para precautelarse a sí mismos, frente a distintas incursiones y presiones en su territorio de movilidad. El 25 de enero de 2016 en el río Shiripuno antes de la confluencia con el río Cononaco Chico se produjo un ataque de un grupo aislado, a una pareja Waorani (Caiga Baihua y Onenka Ñama) de la comunidad de Boanamo, quienes navegaban hacia Baameno, comunidad localizada en la $\mathrm{ZITT}^{42}$. Desde la DPPIAV se compensó a los familiares dolientes en Boanamo, con "6 casas y una malla de protección", sin embargo pueden activarse venganzas, si no se actúa de manera oportuna $^{43}$. La situación en que viven los Waorani de Dicaro, Guiyero y Yarentaro (bloque 16) es

39 Entre los waorani que incursionaron se encontrarían algunos mayores veteranos. Los Wepeiri son un grupo que no fue contactado, ni evangelizado por el ILV. Nunca estuvieron en el protectorado, o estuvieron poco tiempo. Después fueron contactados por Monseñor Labaka.

40 Si bien funcionarios de Repsol, lugareños y capuchinos anticiparon al PMC sobre las incursiones que la familia de los ancianos planificaban, como compra de comida, armas y municiones en el Coca y preparación de guerreros.

41 La Fiscalía de Orellana caldeó los ánimos en las comunidades del bloque 16. En un operativo policial efectuó una incursión en helicóptero para tomar a una de dos niñas PIA tomadas por el grupo que perpetró la venganza de los ancianos, y que permanecía con una de las familias captoras. Fue llevada al hospital para después entregarla a un grupo familiar waorani en Baameno en la ZITT. Se desconoció códigos culturales en que niñas huérfanas son adoptadas como hijas por captores después de un enfrentamiento (Álvarez et al., 2016). También después de sus investigaciones, la Fiscalía encaminó la judicialización de waorani responsables de la incursión; se les acusó de genocidio-etnocidio, para posteriormente procesarles por homicidio. "Fueron apresados 7 guerreros wao de Yarentaro, Dicaro, de los cuales escaparon 2; 5 permaneciendo presos por el lapso de 9 meses; uno de ellos un anciano. Posteriormente fueron liberados por falta de evidencias materiales (cuerpos), argumentándose desde la Corte Constitucional que se iniciaría un tratamiento en el marco del pluralismo jurídico" (Álvarez et al. 2016: 4).

42 La pareja después de haber transportado turistas a Ñoneno, retornaba a Baameno. Como resultado del ataque, Caiga murió atravesado por cuatro lanzas y murió, mientras su esposa Onenka herida en la pierna, logró huir en la canoa.

43 Tomado de: Dos tribus y una vieja rivalidad: vuelven tiempos de guerra a la Amazonía, Soraya Constante (2016). Visitado en https://news.vice.com/es/article/dos-tribus-una-vieja-rivalidad-vuelven-tiempos-de-guerra-a-amazonia. 
álgida y temen acercamientos de PIA. En Tiwuino a mediados del 2015 se registró presencia de PIA por cuatro días, y no funcionó ningún plan de contingencia. Los Waorani temían navegar por los ríos, movilizarse por el territorio e ir a las chacras.

En los lineamientos de la comisión creada en el 2013 se recomienda a la DPPIAV fortalecer el Plan de Medidas Cautelares, incrementar las estaciones de monitoreo, definir intervenciones ante emergencias e intensificar controles en el territorio incluso con intervención disuasiva de la fuerza pública. Se sugiere una política que combine protección de PIA con mitigación de amenazas, y para ello se propone capacitaciones a colindantes Waorani, Kichwas, Shuar, campesinos, técnicos petroleros y otros. Se prevén labores "educativas de los waorani" y como pueblo de reciente contacto, "favorecer su integración plena y digna en sociedad, para que cambien su actitud ante el conflicto", que "superen lógicas de venganza con muertes", y promover una cultura de paz entre Waorani y pueblos aislados ${ }^{44}$.

En definitiva, la DPPIAV se concentraría en pacificar las relaciones entre PIA (TageriTaromenane) y de reciente contacto (Waorani), pero no se establecen medidas para frenar de manera contundente las distintas presiones que ocurren en el Yasuní, principalmente relacionadas a la ampliación de las fronteras extractivas. La política de protección de PIA está subordinada a las políticas del desarrollo, del fomento del extractivismo y capitalización de la naturaleza. En función de esto se delimitó otrora la ZITT y se define desde la institucionalidad, el número de grupos aislados en el Parque Nacional Yasuníit

Al 2008 existía en el Parque Nacional Yasuní actividades extractivas en varios bloques petroleros: el 16 operado por Repsol YPF, el 14 y 17 por Andes petroleum, el bloque 15 operado por Petroecuador, y 31 por Petrobras-PetroEcuador. Al 2016, la ampliación de las fronteras extractivas se ha profundizado. Los pueblos aislados fueron utilizados en imágenes y retórica para apuntalar la Iniciativa Yasuní ITT, que posteriormente fue clausurada, dando paso al plan B de permitir operaciones en los bloques 31 y 43 por parte de PetroAmazonas. Si bien la DPPIAV actualmente indica no registrar señales de presencia de PIA en los bloques 31 y 43; ambientalistas (Acción Ecológica, Colectivo Yasunidos) enfatizan que la actividad petrolera en estos bloques pondrá mayor presión y acorralamiento a grupos en aislamiento ${ }^{46}$.

Otra área problemática para la sobrevivencia de los PIA es el área contigua al campo Armadillo con reservas probadas, ubicado en la parroquia Inés Arango en la provincia de Orellana a pocos kilómetros de la vía Auca. Los registros del PMC cuando tenía sede en el MAE, evidenciaban presencia de PIA en la zona; más recientemente, la DPPIAV también tiene registros de presencia y movilidad, no obstante esto no ha desestimado el inicio de actividades extractivas en parte del campo.

Con las matanzas transcurridas en el siglo XXI, en algo más que una década, seguramente los PIA han sido llevados al borde del exterminio. Enfrentan además la construcción de ejes viales, colonización y extracción forestal ilegal. Hay otras presiones no controladas como el turismo que se hace inclusive dentro de la ZITT ${ }^{47}$. Los enfrentamientos entre Waorani-Tagaeri, WaoraniTaromenane, si bien son pautados por dinámicas de venganza interétnica, están azuzadas por

44 Tomado de Propuesta Técnica y Financiera de consultoría para el Ministerio de Justicia y Derechos Humanos; FEPP. Enero, 2015.

45 En el 2010 el PMC (del MAE) registraba 5 grupos de PIA en el Yasuní; posteriormente en el 2013 cuando clausuraba la Iniciativa Yasuní ITT que consistía en dejar bajo tierra al menos 850 millones de barriles de crudo en el área de los bloques Ishpingo, Tambococha y Tiputini- el PMC con sede en el Ministerio de Justicia anunció que había 4 grupos solamente. Los reubicó en un mapa indicando que se encontraban más al sur. Con ello se daría aval a las actividades de exploración y explotación petrolera en una zona (bloques 31 y 43) donde el anterior mapa evidenciaba registros de presencia de PIA.

46 En un gobierno previo, la empresa brasileña PETROBRAS operó dentro del Parque Nacional Yasuní, y estaba prevista la concesión del bloque 31. Frente a ello ya en el 2007 hubo denuncias y protestas por el riesgo que significaría para los PIA. Actualmente opera PetroAmazonas.

47 Varias comunidades de la ZITT tienen pequeños lodges para llevar turistas y operan con distintas agencias. La actividad puede poner en riesgo a waorani, propiciar enfrentamientos entre Waorani-Tagaeri/Taromenane, y arriesgar la vida de turistas. La decisión de frenar la actividad es compleja, al ser una fuente de ingresos para comunidades waorani. 
las presiones mencionadas. Los lanceamientos y matanzas por ende, no pueden ser explicados exclusivamente en base a dinámicas culturales de tiempos de guerra y venganza endógenas, sino en el contexto de la cronología de eventos de ocupación territorial por parte del Estado y las empresas petroleras.

Como plantean Narváez y Di Marchi (2013), en el Yasuní se superponen políticas territoriales: las de producción de petróleo con concesiones petroleras a empresas nacionales e internacionales, de protección de la naturaleza con las categorías de protección-Parque Nacional y Reserva de Biósfera Yasuní, y el reconocimiento de derechos indígenas, derechos territoriales waorani, ZITT, además de la colonización agraria "que utiliza las vías preparadas para las operaciones petroleras; por ejemplo la vía Auca para la agroindustria (la palma aceitera de Lago Agrio mezclada con las actividades petroleras)" (Narváez y Di Marchi, 2013: 10). Se ha cuestionado los límites de la ZITT por no corresponder a la territorialidad efectiva de los PIA. Colleoni y Proaño (2008) inferían su presencia sobre los ríos Tivacuno, Dicaron, Gabaron y Yasuni, dentro de los bloques petroleros, 14 (entre ríos Tivacuno y Mencaro), 16 (con presencia alrededor de comunidades waorani de Dicaro, Yarentaro, Iro y Gabaron) y campo Armadillo (que involucra a colonos y waorani asentados sobre la carretera). Para Narváez y Di Marchi (2013:14) la ZITT es "una figura artificial", no corresponde a la territorialidad ejercida por los PIA, ni evita que la presión extractivista transnacional condicione las fronteras del territorio indígena, genere más hostilidad y exacerbe la violencia"48.

A1 2016, habrá que esperar si la DIPPIAV realiza una nueva delimitación más aproximada a las dinámicas territoriales de los PIA. Preocupa que las medidas de protección no tengan suficiente efectividad para prevenir encuentros violentos y proteger la vida y continuidad cultural de los PIA. Desde el Estado se da prioridad a garantizar las actividades de la explotación petrolera, con sus efectos en la degradación de la naturaleza y el etnocidio.

Concordamos, con lo que plantea Narváez (2013) de que el Yasuní es un espacio panoptizado. No está suficientemente claro si la ZITT y las políticas para PIA buscan vigilar, controlar y castigar a quienes la transgreden, o más bien ha servido para vigilar y controlar a quienes están dentro de ella.

\section{Conclusiones}

En Brasil la política de protección de PIA se institucionalizó tempranamente en el siglo $\mathrm{XX}$, primero dentro del marco del indigenismo sertanista concentrado en pacificar, proteger, tutelar e integrar, para después desde los 80 asumir una política de no propiciar el contacto. En Ecuador, la creación de una institucionalidad específica de protección a PIA ha ocurrido en el siglo XXI en los últimos 8 años. Ha sido implementada como intento del Estado, de responder a las medidas cautelares otorgadas por la Comisión Interamericana de Derechos Humanos, frente a sucesos ocurridos de enfrentamiento y muerte. Sus lineamientos no han sido la integración, sino virtualmente, la protección de PIA y pacificar las relaciones entre Waorani-Tagaeri/Taromenane.

En ambos países se muestran limitaciones a una efectiva protección de los derechos humanos de los PIA. Se adolece de sintonía entre distintos actores institucionales, y si bien se implementan sofisticados sistemas de monitoreo, que más parecieran panoptizar territorios étnicos, la política pública no se adentra en el foco central del problema. Con esto nos referimos, a que no se frena los frentes económicos (extractivos, agropecuarios, de colonización, entre otros) que provocan intrusiones de distintos actores.

48 Narváez y Di Marchi (2013) enfatizan que la delimitación de la ZITT no responde a los movimientos históricos de los PIA; de lo contrario la misma hubiera afectado no solamente a los bloques 17 (modificado) y 32 (suprimido); sino habría modificado a otros bloques petroleros concesionados en territorio waorani y en el Parque Nacional Yasuní. 
La protección en gobiernos del PT (en el caso de Brasil), de Alianza País (en el caso de Ecuador) en los últimos años, se ve subordinada a nuevas modalidades de integración de la Amazonía con fines de articulación a mercados competitivos. Esto significa mayor capitalización de la naturaleza, mayor presión sobre territorios de pueblos indígenas y mayor acorralamiento de pueblos aislados. Pese a que se han afianzado marcos institucionales y políticas de protección, pareciera que lo que menos interesa a los Estados es precautelar culturas diversas, modos de vida diferentes, ni conservar ecosistemas que son espacios de movilidad y sustento de dichos pueblos.

\section{Bibliografía}

Álvarez, K; Vallejo, I; Bonilla, N; Rodríguez, M y Coba, L. 2016, Conflictividad actual: Ataque de indígenas en aislamiento a familia waorani de Zona Intangible del Parque Nacional Yasuní. Colectivo de Antropólogas del Ecuador, documento entregado al representante residente de las Naciones Unidas en el Ecuador y a la Agregada de Cooperación de la Unión Europea.

2013, Amicus Curiae sobre las medidas cautelares MC-91-06 otorgadas en favor de los pueblos indigenas Tagaeri y Taromenane en situación de aislamiento, Ecuador. Dirigido a CIDH. Colectivo de Antropólogas del Ecuador.

Berraondo, Mikel y Cabodevilla, Miguel Ángel (Eds.) 2005, Pueblos no contactados ante el reto de los derechos humanos: un camino de esperanza para los Tagaeri y los Taromenane, CDES - CICAME, Quito.

Brackelaire, Vincent. 2006, Situación de los últimos pueblos indígenas aislados en América latina (Bolivia, Brasil, Colombia, Ecuador, Paraguay, Perú, Venezuela). Diagnóstico regional para facilitar estrategias de protección, CGII, FUNAI, Belem.

Cabodevilla, Miguel Ángel. 2013, Una tragedia ocultada, CICAME, Quito.

1999, Los Huaorani en la historia de los pueblos del Oriente, Miguel Ángel Cabodevilla, CICAME, Quito.

Ferreira, Saulo Feitosa. 2012, "O isolamiento voluntário e o direito de resistência", en: Loebens, Gunter y Oliveira Neves Lino, Povos indígenas isolados na Amazonia: A luta pela sobrevivencia, Abya Yala, Quito, pp. 43-50.

Foucault, Michel. 2000, Vigilar y castigar: Nacimiento de la prisión, Siglo XXI, México.

1989, Verdad y Poder, La piqueta, Madrid.

Hardt, Michael y Negri, Antonio. 2001, Empire, Harvard University Press.

Huertas, Beatriz. 2002, Los pueblos indigenas en aislamiento. Su lucha por la sobrevivencia y la libertad, Grupo Internacional de Trabajo sobre Asuntos Indígenas (IWGIA), Lima.

Liebgott, Roberto. 2012, "Os povos indígenas em situacao de isolamente e riesco e a política do estado brasileiro para a sua protecao", en: Loebens, Gunter y Oliveira Neves Lino, Povos indigenas isolados na Amazonia: A luta pela sobrevivencia, Abya Yala, Quito, pp. 33- 42.

Loebens, Gunter y Oliveira Neves Lino. 2012, Povos indigenas isolados na Amazonia: A luta pela sobrevivencia, Abya Yala, Quito.

López Fernando y Miranda Arizete. 2012, Povos indigenas isolados na Amazonia: A luta pela sobrevivencia, Abya-Yala, Quito, pp 183-243.

Milanez, Felipe. 2015, Memórias sertanistas: Cem anos de indigenismo no Brasil, SESC, Sao Paulo.

Mindlin, Betty. 2015, Prefacio, en: Memórias sertanistas: Cem anos de indigenismo no Brasil., SESC, Sao Paulo, pp. 11-24.

Ministerio de Medio Ambiente y Unión Europea. 1999, Zonas intangibles de la Amazonía Ecuatoriana, Ministerio de Medio Ambiente, Ministerio de Energía y Minas, CODENPE, PETROECUADOR y Proyecto PETRAMAZ de la Unión Europea.

Ministerio de Justicia y Derechos Humanos. 2015, Propuesta Técnica y Financiera de consultoría para la elaboración e instauración de una política pública de protección a los pueblos en aislamiento voluntario, investigación sobre la realidad y cultura de los PIAV y promoción de una cultura de paz con las poblaciones circundantes en la zona, con la finalidad de prevenir y asegurar la protección de los derechos humanos en la Amazonía ecuatoriana.

2008, Plan de Medidas Cautelares para Pueblos en situación de Aislamiento Voluntario.

Narváez, Iván. 2013, "Los waorani en el Yasuní: contrapoder de los poderes salvajes", en: Narváez, Iván y De Marchi Massimo, Yasuní. Zona de sacrificio. Análisis de la Iniciativa ITT y los derechos colectivos indígenas, FLACSO. Quito pp. 29-55 
2011, "Yasuní y derechos colectivos en el Estado Constitucional de Derechos, intercultural y plurinacional ecuatoriano", en: Krainer, A y Mora M.F (Comp.), Retos y Amenazas en Yasuní, FLACSO-WCS, Quito.

Narváez, Iván y De Marchi Massimo. 2013, Yasuní. Zona de sacrificio. Análisis de la Iniciativa ITT y los derechos colectivos indígenas, FLACSO, Quito.

Proaño, José y Colleoni, Paola. 2008, Taromenane Warani Nani: pueblos indígenas en Aislamiento Voluntario. Tagaeri-Taromenane, en la Amazonía ecuatoriana, Abya Yala, Pachamama, Quito.

Quijano, Aníbal. 2000, "Colonialidad del poder, eurocentrismo y América Latina", en: Edgardo Lander (Comp.) La colonialidad del saber: eurocentrismo y ciencias sociales. Perspectivas Latinoamericanas, CLACSO, Consejo Latinoamericano de Ciencias Sociales, Argentina, Buenos Aires, Julio de 2000, p. 246.

Wallerstein, Immanuel. 1974, The Modern World System I: Capitalist agricultura and the origin of the European World-Economy in the Sixteenth century, Academic press, New York.

Wolf, Erik. 1982, Europe and the People without history, University of California Press, Berkeley. 